\title{
O Construcionismo e o software de programação App Inventor 2 na formação inicial de professores de Ciências
}

\author{
Rafael Felipe Pszybylski - IFPR, rafael.pszybylski@ifpr.edu.br \\ Marcelo Souza Motta - UTFPR, msmotta27@gmail.com \\ Marco Aurélio Kalinke - UTFPR, marcokalinke@yahoo.com.br
}

Resumo. Este trabalho tem por objetivo analisar se o desenvolvimento de aplicativos educacionais móveis no software de programação App inventor 2, possibilita a criação de um ambiente de aprendizagem construcionista na formação inicial de professores de Ciências. A pesquisa foi realizada com estudantes do curso de Licenciatura em Ciências Biológicas de uma instituição pública do Paraná. A apresentação do software ocorreu por meio de um guia didático introdutório, que aborda as principais funções do App Inventor, a construção detalhada de uma calculadora, um jogo no formato de quiz e alguns desafios. Após a aplicação do guia didático, os estudantes construíram aplicativos educacionais móveis autorais para o ensino de Física. A metodologia da pesquisa se refere a uma abordagem qualitativa e para a constituição dos dados, utilizaram-se diferentes instrumentos metodológicos, a saber: observações, anotações, questionários, relatórios e os aplicativos desenvolvidos pelos estudantes. A análise dos dados revelou que o App inventor 2 permitiu a criação de um ambiente de aprendizagem construcionista, potencializando situações de ensino e aprendizagem criativas, inovadoras e reflexivas.

Palavras-chave: Formação Inicial de Professores. Tecnologias Digitais na Educação. App inventor. Dimensões do Construcionismo. Aplicativos Educacionais Móveis

\section{The Constructionism and the programming software App Inventor 2 in the initial teacher education}

\begin{abstract}
This work aims to analyze whether the development of mobile educational applications in the App inventor 2 programming software, allows the creation of a constructionist learning environment in the initial training of Science teachers. The research was carried out with undergraduate students in the Biological Sciences course at a public institution in Paraná. The software was presented through an introductory didactic guide, which covers the main functions of the App Inventor, the detailed construction of a calculator, a quiz game and some challenges. After applying the didactic guide, students built authoritative mobile educational apps for teaching Physics. The research methodology refers to a qualitative approach and for the constitution of the data, different methodological instruments were used, namely: observations, notes, questionnaires, reports and the applications developed by the students. Data analysis revealed that App inventor 2 allowed the creation of a constructionist learning environment, enhancing creative, innovative and reflective teaching and learning situations.
\end{abstract}

Keywords: Initial Teacher Education. Digital Technologies in Education. App inventor. Dimensions of Constructionism. Mobile Educational Apps. 


\section{Introdução}

Uma temática bastante atual em pesquisas relacionadas à formação inicial e continuada de professores, é o uso das tecnologias digitais de informação e comunicação (TDIC) direcionadas aos processos de ensino e aprendizagem. Kenski (2012) expõe que essas ferramentas podem proporcionar inovações nas concepções educacionais, novas possibilidades de interação e de comunicação e formas diferenciadas de aprendizagem.

Neste contexto, surge a necessidade de investigar maneiras de criar ambientes de aprendizagem que possibilitem aos professores e licenciandos a reflexão, a crítica e o protagonismo na utilização das TDIC. Lima e Loureiro (2019) defendem a ideia de que cursos de formação devem proporcionar aos professores a construção de Materiais Autorais Digitais Educacionais (MADE) que se caracterizam como uma produção planejada, desenvolvida e executada por meio de softwares digitais, com finalidade de auxiliar a mediação dos processos de ensino, aprendizagem e avaliação (LIMA; LOUREIRO, 2016).

Nesta perspectiva, o software de programação App Inventor 2 se constitui como uma ferramenta que possibilita a construção de MADE por meio do desenvolvimento de aplicativos para dispositivos que operam com o sistema operacional Android. Entre os fatores que justificam a escolha deste software estão a sua gratuidade e o fato de não haver necessidade de conhecimentos aprofundados de programação para o desenvolvimento dos aplicativos, pois a estruturação do programa é realizada por meio de blocos, que exploram as ideias de uma programação intuitiva e visual.

Segundo Elias (2018), com App Inventor 2 é possível criar aplicativos personalizados, de acordo com o interesse do desenvolvedor, de forma livre e gratuita. Assim, professores não ficam restritos aos aplicativos já disponíveis nas lojas virtuais, podendo criar seus próprios MADE com características personalizadas que contemplem os seus objetivos educacionais.

Para fundamentar e avaliar pesquisas que visam à produção de MADE é necessário uma teoria educacional coerente com essa proposta. Neste sentido, a teoria da aprendizagem denominada por Construcionismo, criada por Seymour Papert, ganha destaque pela integração das TDIC ao processo de ensino. Na concepção de Papert (1994), os computadores podem e devem ser utilizados como ferramentas para trabalhar e raciocinar, como meios de criação e realização de projetos, como fonte de inspiração para pensar novas ideais e não apenas como uma forma de apoio à instrução automatizada.

Diante do exposto, este trabalho tem por objetivo analisar como o desenvolvimento de aplicativos educacionais móveis, utilizando o software de programação App inventor 2, possibilita a construção de um ambiente de aprendizagem construcionista na formação inicial de professores de Ciências.

\section{O Contrucionismo}

Papert (1986) apresenta duas ideias centrais que se fazem presentes em situações de aprendizagem baseadas no Construcionismo. Primeiro, o estudante precisa construir alguma coisa, ou seja, é o aprendizado por meio do fazer, colocando algo em prática. Segundo, é o fato de que o estudante precisa construir um produto de significado pessoal e para o qual está motivado, como uma obra de arte, um relato de experiência ou um programa de computador (VALENTE, 1993) 
Neste sentido, o uso das TDIC no paradigma construcionista visa possibilitar a criação de situações que envolva a participação ativa do estudante, valorizando seus pensamentos, a expressão de ideias e a construção de um artefato tecnológico de seu interesse. No caso da formação de professores, o processo de construção de MADE, utilizando uma linguagem de programação, permite a criação de produtos concretos e personalizados, fazendo o uso da criatividade, o que auxilia na reorganização do pensamento e cria oportunidades de construção de conhecimento por meio do ciclo de aprendizagem destacado por Valente (1993; 2006), a saber: descrição, execução, reflexão e depuração.

A descrição se inicia com a escrita dos procedimentos necessários para a construção do que se está planejando, utilizando para isso uma linguagem de programação. O processo de execução ocorre quando o aprendiz acompanha a realização dos passos planejados na programação, sendo executado no computador ou aparelho similar.

O processo de reflexão ocorre por meio da análise da resposta obtida na execução do programa, se tudo estiver correto, o objetivo da atividade foi alcançado; em caso de erro, o estudante poderá repensar sua estratégia realizando a abstração empírica e pseudo-empírica (VALENTE, 1993). A abstração empírica é o nível de abstração mais simples, que possibilita ao aluno extrair informações de um objeto, tais como, cor, dimensão e a sua forma. A abstração pseudo-empírica tem um grau mais alto de complexidade e permite ao aluno relacionar a ação de um objeto com a programação realizada na fase da descrição.

A partir do resultado recebido, o estudante pode passar pelo processo de depuração de sua ideia, identificando acertos e erros, promovendo, se for necessário, novas estratégias na programação até que esteja satisfeito com a resposta obtida. O ciclo descrição-execução-reflexão-depuração-descrição pode ocorrer seguidas vezes até que o resultado desejado seja encontrado, proporcionando, assim, o refinamento de ideias, tornando o aluno agente ativo na construção de sua própria aprendizagem (VALENTE, 1993).

Desta maneira, as ideias construcionistas integradas às atividades de programação possibilitam aos estudantes a construção de conhecimentos de forma ativa, contínua e que se reforçam (VALENTE, 2016).

Sápiras, Vecchia e Maltempi (2015) expõem que mesmo que as TDIC desempenhem um papel de destaque na concepção construcionista, o desenvolvimento de um ambiente de aprendizagem efetivo não é garantido com a simples inserção de uma ferramenta tecnológica. Em vista disso, Papert (1986) por meio da análise de diversos estudos, definiu cinco dimensões que servem para fundamentar $o$ desenvolvimento de ambientes de aprendizagem baseados no Construcionismo, conforme evidencia Maltempi (2004):

I) Dimensão pragmática: evidencia a importância de que o conteúdo deve ser utilizado para um fim prático, o estudante precisa construir algo útil para o momento em que está vivendo, de forma que o artefato desenvolvido seja utilizado de imediato. Com isso, o estudante tem a sensação de praticidade e poder.

II) Dimensão sintônica: transmite ao estudante uma relação de proximidade com o conteúdo abordado. Para tanto, o aluno precisa ser um agente ativo e participar da escolha do tema, fazendo com que o projeto se torne mais relevante. Isto facilita a relação entre aprendiz e projeto, aumentando as chances de o conteúdo abordado ser mais bem assimilado;

III) Dimensão sintática: refere-se à facilidade com que o estudante acessa conhecimentos básicos e avança nos seus estudos usando o seu desenvolvimento 
cognitivo, sem a necessidade de pré-requisitos. Para isso é importante à utilização de matérias acessíveis aos conhecimentos prévios do estudante.

IV) Dimensão semântica: permite ao estudante interagir com elementos que tenham significado para si, ou seja, possibilita a manipulação e o trabalho com ferramentas que são de seu interesse, motivando-o para descobrir novos conceitos e aplicações, sem que a aprendizagem carregue meros formalismos e símbolos;

V) Dimensão social: traz a atividade ao cotidiano do estudante, de modo que o aprendizado seja construído em conjunto com as relações pessoais, contribuindo para sua valorização social e a integração cultural do ambiente em que está inserido.

Sobre estas dimensões, Sápiras, Vecchia e Maltempi (2015), argumentam que quando estimuladas podem facilitar o processo de construção de conhecimento dos estudantes, pois leva em consideração seus interesses, seus conhecimentos prévios e seu posicionamento frente a realidade social e cultural.

Em vista do exposto, verifica-se que o software de programação App Inventor 2 , pode ser considerado uma tecnologia apropriada para uma abordagem Construcionista, uma vez que faz uso de uma linguagem de programação que permite a criação de aplicações realizando o ciclo destacado por Valente $(1993 ; 2016)$ à medida que exercita a criatividade, tornando o aprendizado mais lúdico e interativo. Além disso, possibilita a construção do ambiente de aprendizagem que valoriza o fator social e cultural do aprendiz, estimulando discussões de ideias e projetos a partir das cinco dimensões apresentadas.

\section{Metodologia}

A pesquisa apresenta uma abordagem qualitativa, pois, os pesquisadores estiveram em contato direto e prolongado com o ambiente investigado. Além disso, a ênfase na constituição dos dados foi dada ao processo, ao invés dos resultados ou produtos. Deste modo, buscam-se informações mais descritivas, que primam pelo significado dado às ações (BORBA; ARAÚJO, 2012).

A turma em que foi aplicada a proposta era composta por 23 estudantes do curso de Licenciatura em Ciências Biológicas do Instituto Federal do Paraná (IFPR) Campus Palmas. Ao total foram realizados sete encontros, com duração de quatro horas cada. Para apresentar o software App inventor 2, foi aplicado nos três primeiros encontros, um guia didático ${ }^{1}$ introdutório, que comtempla a apresentação dos principais recursos, atividades práticas de desenvolvimento de aplicativos e desafios.

No primeiro encontro, os estudantes foram expostos aos objetivos da pesquisa e à metodologia que seria utilizada. Na sequência, apresentou-se o software de programação App inventor 2, como ele pode ser utilizado, suas principais funções, a tela de designer do aplicativo, a tela de programação e os blocos lógicos.

Com o auxílio do guia didático, no segundo encontro, exploraram-se as funções básicas de programação por meio da construção detalhada de uma calculadora que soma dois números digitados pelo usuário e, em seguida, foi proposto o desafio de acrescentar à programação as operações de subtração, divisão, multiplicação e divisão. Esta atividade objetivou evidenciar os recursos disponíveis na área de programação, como os blocos de variáveis, matemática e controle.

O terceiro encontro foi destinado à programação de um jogo no formato de quiz, com perguntas sobre o conteúdo de Termodinâmica. Esta atividade visou

\footnotetext{
${ }^{1}$ Propostas de atividades utilizando o Software de programação App Inventor 2 em um ambiente de aprendizagem construcionista.

$<\underline{\text { http://repositorio.utfpr.edu.br/jspui/bitstream/1/4622/2/CT_PPGFCET_M_Pszybylski\%2c_Rafael_Felip }}$ e 2019 1.pdf $>$
}

V. $18 \mathrm{~N}^{\mathrm{o}}$ 1, julho, 2020 RENOTE

DOI: 
apresentar as funções mais sofisticadas de programação, como mudança de tela e opção de seleção de respostas. Após a sua programação, foi proposto um desafio para aperfeiçoá-lo, a fim de mostrar ao estudante a resposta correta, caso ele selecionasse a alternativa errada.

Após a aplicação do guia didático, os estudantes se reuniram em grupos e iniciaram a construção de seus aplicativos educacionais autorais, utilizando para isso três encontros seguintes. Foi proposto que fosse desenvolvido aplicativos com temas relacionados ao ensino de Física.

No último encontro, os aplicativos educacionais construídos pelos estudantes foram apresentados a todos os participantes da pesquisa de forma a socializar suas ideias. Também foi aplicado o questionário final de pesquisa, com questões que buscavam investigar como foi o processo de criação dos aplicativos, como os alunos avaliavam o App inventor 2, se o curso contribuiu para a sua formação inicial e a avaliação final da pesquisa.

Para responder aos objetivos traçados em uma pesquisa que adota a abordagem qualitativa, Borba e Araújo (2012) sugerem utilizar múltiplos procedimentos para obtenção de dados, como uma forma de aumentar a confiabilidade e a credibilidade da constituição dos dados. Portanto, nesta pesquisa, foram utilizadas como procedimentos metodológicos: observações, anotações, diário de campo, questionários e relatórios, com os pesquisadores acompanhando, sistematicamente, todas as etapas do desenvolvimento dos projetos. O Quadro 1 apresenta os instrumentos metodológicos utilizados em cada encontros.

Quadro 1 - Organização dos Encontros

\begin{tabular}{|c|l|l|}
\hline Encontro & \multicolumn{1}{|c|}{$\begin{array}{c}\text { Instrumentos Metodológicos } \\
\text { Utilizados }\end{array}$} & \multicolumn{1}{c|}{ Atividade Realizada } \\
\hline $1^{\circ}$ & $\begin{array}{l}\text { Questionário de entrada; Observações } \\
\text { e Anotações. }\end{array}$ & $\begin{array}{l}\text { Apresentação da pesquisa; Apresentação App } \\
\text { Inventor. }\end{array}$ \\
\hline $2^{\circ}$ & Relatório; Observações e Anotações. & Construção de uma calculadora simples. \\
\hline $3^{\circ}$ & Relatório; Observações e Anotações. & Construção de um quiz. \\
\hline $4^{\circ}$ & Relatório; Observações e Anotações. & Confecção dos aplicativos. \\
\hline $5^{\circ}$ & Observações e Anotações. & Confecção dos aplicativos. \\
\hline $6^{\circ}$ & Observações e Anotações. & Confecção dos aplicativos. \\
\hline $7^{\circ}$ & $\begin{array}{l}\text { Observações; Anotações e } \\
\text { Questionário Final. }\end{array}$ & Apresentação dos aplicativos. \\
\hline
\end{tabular}

Fonte: Elaborado pelo Autor, 2020.

Para possibilitar uma melhor análise dos dados, todo material obtido durante a realização da pesquisa foi organizado e categorizado, com o objetivo de identificar tendências e padrões relevantes que possibilitem obter informações que respondam aos objetivos da pesquisa.

\section{Resultados e Discussão}

Os estudantes foram divididos em 11 grupos que, para efeito de análise dos dados constituídos e preservação da identidade, foram identificados por letras maiúsculas, de $\mathrm{A}$ até $\mathrm{K}$. Os estudantes também foram categorizados individualmente como A1 e A2 do grupo A; B1 e B2 do grupo B; e assim sucessivamente. Como havia 23 estudantes e 11 grupos, o grupo I foi composto de 3 estudantes, identificados como I1, I2 e I3. Ressalta-se que, nas citações dos estudantes, ao longo do texto, não foram corrigidos eventuais equívocos de Língua Portuguesa, transcrevendo na íntegra as respostas dadas. 
No primeiro encontro, antes do início da apresentação do software App inventor 2 , os estudantes responderam um questionário que teve por objetivo identificar seus conhecimentos sobre linguagens de programação, sobre o software em questão, suas concepções em relação ao uso das TDIC nos processos de ensino e aprendizagem e suas expectativas em relação às atividades propostas.

A respeito de seus conhecimentos acerca das TDIC nos processos de ensino aprendizagem, 11 estudantes responderam que possuem conhecimentos básicos, oito afirmaram que seus conhecimentos se limitam a apresentação de slides e uso de notebook. Apenas quatro estudantes apresentavam indícios de ter utilizado as TDIC em atividades de ensino de maneira mais efetiva, como relata a estudante (E1) "Utilização de aplicativos com QR code para realizar atividades como jogos".

Em relação ao conhecimento sobre linguagens de programação e o software de programação App inventor 2, todos os estudantes responderam que não possuíam experiência com este tipo de programação, tampouco utilizaram anteriormente $o$ software App Inventor 2.

Quanto às concepções dos estudantes em relação ao uso das tecnologias nos processos de ensino e aprendizagem, todos consideram importante a sua inserção. Para eles, o uso das TDIC aproxima o ambiente escolar à realidade do aluno "[...] contribui para melhorar o aprendizado dos alunos, este que vive inserido no mundo tecnológico"(D2). Além disso, comentam sobre as "batalhas" que os professores enfrentam ao utilizar as tecnologias em escolas públicas "O Apoio da tecnologia sempre é bom, quando é usada de forma correta, mas temos muitas batalhas ainda principalmente nas escolas públicas” (I3).

No que se refere às expectativas dos estudantes a respeito das atividades a serem realizadas com o App Inventor 2, a análise do questionário revelou que a proposta foi bem recebida, eles se mostraram motivados a adquirir novos conhecimentos e a utilizá-los em sua prática docente "Como professora, espero que o desenvolvimento de aplicativos me proporcione novas metodologias de ensino, inclusive trabalhando com o lúdico como jogos que podem ser criados pelo professor"'(D2).

Portanto, apesar do pouco conhecimento em relação ao uso das tecnologias no ambiente educacional e o desconhecimento a respeito do App inventor 2 e das linguagens de programação, os estudantes consideraram que a atividade proposta pode de alguma forma ser útil à atividade docente, visto que todos entendem, em algum grau de importância, que as TD proporcionam um ambiente em sala de aula mais rico e conectado a realidade dos alunos. Desta forma, foi possível identificar a presença da dimensão pragmática, que versa sobre a importância do aluno aprender algo que possa ser utilizado para algum fim prático.

O guia didático possibilitou acesso e progressão no ambiente de trabalho do software App inventor 2, começando com as funções mais simples, em nível introdutório, para um nível mais alto de complexidade em relação à atividade anterior "No início do curso ainda não estávamos familiarizados com as possibilidades que o app inventor possui [...] com o passar das aulas aprendemos as funções que ele possui e começamos a criar nosso próprio aplicativo" (E2). Desta forma, os estudantes, aos poucos, foram se familiarizando com a linguagem do software "foi um material bem explicativo, com o seu passo a passo nos auxiliou e tornou mais fácil a compreensão para o uso adequado do software" (H2).

Considerando estes relatos e as observações dos pesquisadores, é possível afirmar que o guia didático auxiliou os alunos na superação das dificuldades encontradas com a programação, bem como possibilitou acesso a conhecimentos 
básicos e progressão sem a necessidade de conhecimentos prévios o que evidencia a presença de elementos da dimensão sintática.

Em relação à avaliação dos estudantes a respeito do software App Inventor 2, verificou-se que a possibilidade de utilizar diversos elementos gráficos (cores, figuras, vídeos etc.), que definem as características estéticas do aplicativo e a liberdade em manipular componentes que lhes fazem sentido no ambiente de aprendizagem, foi um aspecto muito elogiado "Possibilita várias opções para moldar o aplicativo conforme a ideia e criatividade do aluno" (C2). Deste modo, eles puderam utilizar múltiplos elementos para apresentar determinado conceito sem a necessidade de conhecimentos aprofundados de programação "É uma ferramenta muito interessante, fácil e prática de ser utilizada, que não precisa ter conhecimentos avançados para configurar os aplicativos" (C2). Tais considerações demonstram a importância da presença da dimensão semântica.

Durante o processo de desenvolvimento dos aplicativos, os estudantes reconheceram que o App Inventor 2 pode contribuir para práticas inovadoras de ensino, "O software possibilita que venhamos trazer novas formas de aprendizado para os alunos, pois ele possui várias ferramentas que auxiliam na criação de um app" (G2). Também, consideraram que, com mais tempo e dedicação ao software, seria possível construir aplicativos mais elaborados que permitissem maior interatividade com o usuário, como a criação de jogos educativos. A oportunidade de autoria gerou nos estudantes o sentimento de construir algo útil que poderá ser utilizado em sua futura prática docente "A criação em sala foi produtiva, acredito que futuramente possa ser utilizado para minha prática docente" (B2). Nesta perspectiva, verificou-se uma relação de proximidade dos estudantes com o software App Inventor 2 "Possui um caráter simples de funcionamento, sendo super fácil para montar as atividades e o seu designer"'(A1). Com isso, foi possível perceber indícios que a pesquisa promoveu a dimensão sintônica.

Mesmo a linguagem de programação do App Inventor 2 sendo simples e intuitiva, os sujeitos relataram que a fase mais complexa foi pensar na lógica de programação. Dar os comandos para o computador do que era para ser executado, por meio de comandos, não foi trivial, alguns não sabiam como avançar em certas situações, precisando recorrer a ajuda do pesquisador, a tutoriais na internet ou aos colegas " $O$ processo de criação do aplicativo foi de muita aprendizagem, tivemos alguns problemas, porém buscamos a solução com o auxílio do professor e vídeos no Youtube" (A2). Valente (1993) argumenta que não basta colocar o estudante diante de um computador, é necessário que haja a mediação do professor, que será responsável por esclarecer as dúvidas que irão surgir, dialogando e estimulando os alunos nos momentos em que surgirem as dificuldades. O ambiente de pesquisa estimulou colaboração e a troca de experiências, evidenciando a presença da dimensão social para que as dificuldades fossem superadas.

As observações do pesquisador e os relatos dos estudantes indicam que eram nos momentos de dificuldades que os participantes refletiam sobre os resultados que apareciam na tela, comparando com o que haviam planejado. Caso não estivesse de acordo com o projeto, corrigiam erros, mudavam as estratégias, adaptavam ideias e buscavam soluções diferenciadas. Isso possibilitou ao professor de Ciências, em formação inicial, experimentar e vivenciar o processo de elaboração e criação de aplicativos educacionais móveis, envolvidos no ciclo reflexivo de aprendizagem descrição, execução, reflexão e depuração presente na atividade de programação (VALENTE, 1993; 2016). 
Apesar das dificuldades relatadas, a tarefa de desenvolver aplicativos educacionais foi cumprida com sucesso por todas as equipes com diferentes habilidades no uso das TD. A análise do questionário final revelou que a possibilidade de construir aplicativos gerou um misto de sentimentos, que foram desde a satisfação pessoal, em conseguir desenvolver algo próprio, ao reconhecimento de suas limitações quanto ao uso de tecnologias "O curso foi excelente e contribuiu para minha formação pois ainda não tinha entrado em contato com programas como este e sei que futuramente poderei utilizar para preparar aulas diferentes para aplicar aos alunos" (B2).

No último encontro, em que foi realizada a troca de aplicativos entre as equipes, os estudantes passaram por um processo de reflexão e discussão sobre a sua própria formação. Neste momento, ocorreu um debate sobre a necessidade de buscar por conhecimentos e recursos que apoiem a sua atuação profissional futura, conforme destaca o estudante H2: "Durante o curso foi possível perceber o quão devemos nos especializar e atualizar". Além disso, refletiram sobre como adequar suas estratégias de acordo com as necessidades de seus alunos e os suportes tecnológicos que tenham a disposição, conforme relata a estudante E2: "O curso fez com que eu pense no meu futuro como professora, pense em como inovar e fazer a diferença nas escolas".

Vale salientar que, a dificuldade com a linguagem de programação e com o conteúdo de Física foi um fator comum entre alguns estudantes. Contudo, a maioria deles, mesmo com estas dificuldades, procuravam participar ativamente das atividades, buscando informações na internet, interagindo com o pesquisador e com os demais colegas a fim de superar os desafios impostos pela pesquisa. Em contrapartida, alguns estudantes, mesmo com dificuldades, não apresentavam interesse em mudar sua condição, realizando o mínimo para conseguir realizar as tarefas propostas. Apesar disso, todos os grupos conseguiram êxito na construção das atividades propostas no guia didático e no aplicativo autoral.

Em relação aos aplicativos construídos pelos estudantes, foi possível identificar o uso de recursos que promovem a interatividade com o usuário, como a criação de quiz, uso de imagens, gifs e links para vídeos na internet. No entanto, alguns deles apresentam limitações em relação à apresentação dos conteúdos, visto que a participação do usuário se limita a leitura de textos informativos, privilegiando o processo de transmissão de conhecimento, apesar a pesquisa evidenciar aspectos construcionistas.

Os aplicativos construídos podem ser acessados na galeria do software app inventor 2, buscando pelos seguintes títulos: "Eletrostática Grupo A", "Eletrostática Grupo B”, “TermoFísica Grupo C”, “Acústica Grupo D”, “Ótica Grupo E”, “Energia Mecânica Grupo F”, "Fluídos Grupo G”, "Leis de Newton Grupo H”, "Densidade Grupo I", "Ondas Grupo J" e "Gravitação Grupo K”.

\section{Considerações Finais}

A teoria de aprendizagem construcionista, proposta por Papert (1986), foi o principal aporte teórico desta investigação, servindo como fundamentação para a preparação do ambiente de pesquisa e para a análise dos dados.

Este estudo permitiu concluir que as cinco dimensões construcionistas foram evidenciadas pelo conjunto: software App inventor 2, guia didático e ambiente de pesquisa. As dimensões pragmática e social foram potencializadas pelo ambiente de pesquisa construído pelo pesquisador em conjunto com os participantes. As dimensões sintática e semântica foram evidenciadas pelas possibilidades de interatividade com o software App inventor 2. A dimensão sintônica surgiu por meio da aplicação do guia didático, que permitiu acesso ao software de forma mais simples possível a todos. 
Cabe destacar que a pesquisa tentou sempre proporcionar um ambiente construcionista, ou seja, acolhedor, motivador e dinâmico. Também incentivou momentos em que ocorriam trocas de conhecimentos entre os grupos, mantendo, assim, uma conexão entre os participantes da pesquisa, valorizando momentos de reflexão sobre suas limitações em relação ao uso das TD na realização do ciclo de programação descrição, execução, reflexão e depuração, bem como a necessidade de aprimorar o uso de ferramentas que podem apoiar a futura prática docente.

Desta forma, esta pesquisa defende que a simples inserção das TDIC nos processos de ensino e aprendizagem, como uma nova forma de transmitir conhecimento, não é inovar e nem traz mudanças significativas nas práticas tradicionais de ensino. É preciso, então, incorporá-las como meios para construção e mediação da própria prática educacional, sabendo identificar as suas limitações e investigando as mais adequadas formas de incluí-las pedagogicamente. Dar condições para o professor pensar criticamente o uso das TDIC, além de torná-lo um agente produtor de MADE e não mero consumidor, pode lhe garantir a segurança para, com conhecimento de causa, incorporá-las em sua prática com qualidade.

Por fim, o software de programação App inventor 2 potencializou situações de ensino e aprendizagem criativas, inovadoras e reflexivas. O seu uso, permitiu a construção de um ambiente de aprendizagem construcionista na formação inicial de professores de Ciências. Como sequência deste estudo, indica-se a realização de pesquisas futuras que busquem evidências das dimensões contrucionistas em outros artefatos tecnológicos e como elas impactam no ambiente escolar.

\section{Referências}

BOGDAN, R.; BIKLEN, S. Investigação qualitativa em educação: uma introdução à teoria e aos métodos. Portugal: Porto Editora, 1994.

BORBA, M. C.; ARAÚJO, J. L. (org.). Pesquisa Qualitativa em Educação matemática. 5. ed. Belo Horizonte: Autêntica, 2012.

ELIAS, A. P. de A. J. Possibilidades de utilização de smartphones em sala de aula: construindo aplicativos investigativos para o trabalho com Equações do $2^{\circ}$ Grau. 2018. Dissertação (Mestrado em Ensino de Ciências e Matemática) - Universidade Tecnológica Federal do Paraná, Curitiba, 2018.

KENSKI, V. M. Educação e tecnologias: o novo ritmo da informação. Campinas: Papirus, 2012.

LIMA, L. de; LOUREIRO, R. C. Integração entre Docência e Tecnologia Digital: o desenvolvimento de Materiais Autorais Digitais Educacionais em contexto interdisciplinar. Revista Tecnologias na Educação, Fortaleza, v.17, n.8, p.1-11, 2016.

LIMA, L de; LOUREIRO, R. C. A compreensão de licenciandos sobre a utilização das tecnologias digitais na docência: um estudo de caso. Informática Na Educação: teoria e prática, Porto Alegre, v. 22, n. 3, 2019.

MALTEMPI, M. V. Construcionismo: pano de fundo para pesquisas em informática aplicada à educação matemática In: BICUDO, M. A. V.; M. C. BORBA (Orgs.).

Educação Matemática: pesquisa em movimento. São Paulo: Cortez, 2004.

PAPERT, S. A Máquina das Crianças: Repensando a Escola na Era da Informática.Porto Alegre: Artmed Editora, 1994.

PAPERT, S. Constructionism: a new opportunity for elementar Science educaction. Massachusetts Institute os Technology, The Epistemology And Learning Group, 1986. 
SÁPIRAS, F. S.; VECCHIA, D. R.; MALTEMPI, M. V. Utilização do Scratch em sala de aula. Educação Matemática Pesquisa: Revista do Programa de Estudos Pós-Graduados em Educação Matemática, v. 17, n. 5, 2015.

VALENTE, J. A. Computadores e Conhecimento: repensando a educação. Campinas, SP: UNICAMP/NIED, 1993.

VALENTE, J. A. Integração do Pensamento Computacional no Currículo da Educação Básica: Diferentes Estratégias Usadas e Questões de Formação de Professores e Avaliação do Aluno. Revista e-Curriculum, v. 14, n. 3, 2016. 